Ann. Sci. forest., 1977, 34 (1), 1-16.

\title{
Recherche des meilleures conditions d'enracinement des boutures herbacées de chêne rouvre (Quercus petraea (M.) Liebl.) et de hêtre (Fagus silvatica L.)
}

\author{
C. CORNU*, S. DELRAN**, J. GARBAYE**, F. LE TACON** \\ avec la collaboration technique de : \\ D. BOUCHARD **, F. BOURGEOIS **, Pierrette CAPELLI * \\ J. COUPAYE * et G. COURRIER ** \\ * Station d'Amélioration des Arbres forestiers, I.N.R.A. Centre de Recherches forestières d'Orléans \\ Ardon, 45160 Olivet \\ ** Station de Recherches sur les Sols forestiers ef la Fertilisation C.N.R.F. I.N.R.A. \\ Champenoux, 54280 Seichamps
}

\section{Résumé}

La multiplication végétative du chêne et du hêtre permettrait de faire progresser les techniques de régénération artificielle et d'améliorer la qualité des peuplements de ces deux essences en France.

Après deux années de recherche sur le bouturage herbacé du chêne et de hêtre, nous avons obtenu les résultats suivants (boutures prélevées en mai-juin, bouturage en serre, avec brumisation pendant 3 mois environ) :

- il est possible d'obtenir 80 à 90 p. 100 de boutures racinées de chêne ou de hêtre, soit à partir de jeunes plants en pépinière, soit à partir d'arbres adultes en utilisant les rejets de souche après abattage ;

- les meilleurs résultats sont obtenus par poudrage du bas de la bouture avec du talc contenant 0,5 p. 100 d'AlB et 15 p. 100 de Benomyl (fongicide) ;

- l'aptitude au bouturage d'un plant est encore excellente à 5 ans, et le recépage du plant ne compromet pas l'aptitude des pousses au bouturage ;

- la tourbe pure est un bon substrat de bouturage.

\section{Introduction}

Face à l'évolution des besoins de la sylviculture du Chêne et du Hêtre en France, la multiplication végétative semble une technique prometteuse.

Elle permettrait en effet de faciliter et d'améliorer les techniques de régénération artificielle en apportant des solutions neuves à différents problèmes :

- rareté et irrégularité des fructifications,

- difficultés de conservation des glands,

- création de peuplements produisant du bois de haute qualité technologique. 
Dans le domaine de la recherche, la multiplication végétative du chêne et du hêtre permettrait d'expérimenter sur un matériel clonal présentant une bonne homogénéité génétique. Enfin, elle constituerait une alternative au greffage pour la conservation de génotypes ef la constitution de vergers à graines.

Nous avions le choix entre différentes techniques de multiplication : bouturage (boutures dormantes ou herbacées) ou multiplication in vitro (à partir de cals, d'apex ou de méristèmes).

Nous avons choisi de commencer par le bouturage, en espérant ainsi mettre au point rapidement une technique simple, efficace et fiable.

L'expérience acquise pour ces deux essences réputées souvent, mais à tort, rebelles au bouturage est rare (Muhle-Larsen, 1946 ; Thimann et Behnke-Rogers, 1950 ; Flemmers, 1962) mais permet de dégager les certitudes suivantes :

- le bouturage à partir de rameaux dormants est irréalisable,

- le bouturage à partir de pousses non aoûtées prélevées au printemps (« boutures herbacées ») peut donner de bons résultats, à condition de n'utiliser que du matériel physiologiquement jeune - les pousses prélevées dans la cime d'un arbre adulte donnent de très mauvais résultats - et d'assurer sa survie jusqu'à la formation des racines.

A la suite d'une première expérience à petite échelle réalisée en 1974, nous avons montré dans une courte note (Cornu, Garbaye, Le Tacon, 1975) qu'il était facile d'obtenir des forts taux d'enracinement pour le chêne et le hêtre. Ces premiers résultats nous ont conduit à mettre en jeu un plus grand nombre de facteurs en 1975 . Entre temps, Kleinschmit, Witte et Sauer (1975) ont fait état de leurs premiers résultats sur le chêne en Allemagne. Nous en tiendrons compte dans la discussion. Nous exposerons en détail nos propres résultats obtenus en 1974 et 1975 à la fois à partir de jeunes plants, pouvant jouer le rôle de pieds-mères pour la multiplication massive en pépinière, et à partir de rejets de souche d'arbres adultes pouvant constituer une tête de clone.

Dans un premier temps, nous n'étudierons que l'enracinement des boutures, sans tenir compte de leur croissance ultérieure et de l'élevage des plants ainsi obtenus.

\section{Matériel et méthodes}

Les conditions expérimentales décrites ici concernent la majorité des essais. Les exceptions seront signalées lors de l'exposé des résultats.

\subsection{Matériel végétal}

En 1974, les rejets de souche de chêne rouvre (Q. petraea Liebl.) et de hêtre (Fagus silvatica L.) ont été prélevés dans une parcelle de la forêt domaniale d'Amance (Meurthe et Moselle) de 60 ans environ, coupée à blanc pendant l'hiver précédent.

Les boutures de jeunes plants provenaient de semis naturels de 5 ans environ, en forêt de Champenoux (Meurthe et Moselle) pour le chêne, et en forêt de Haye (Meurthe et Moselle) pour le hêtre.

En 1975, les rejets de souche provenaient d'une éclaircie réalisée l'hiver précédent dans une futaie de 90 à 100 ans en forêt de Champenoux, et les rejets de hêtre de la parcelle déjà utilisée en 1974 (seuls les nouveaux rejets ont été prélevés). 
Les boutures de jeunes plants provenaient de semis vigoureux de 1 an, élevés sur tourbe fertilisée en pépinière (provenance : Champenoux pour le chêne et Roumanie pour le hêtre).

\subsection{Prélèvement et préparation des boutures}

Dans presque tous les essais réalisés, les boutures sont constituées par l'extrémité des pousses avec le bourgeon terminal. Le prélèvement a lieu de début mai à fin juin, suivant le stade de développement : dès que la pousse présente une rigidité suffisante. La détermination du stade cptimal de développement fera l'objel d'expériences en 1976.

Dans le cas des jeunes plants, le prélèvement a lieu indifféremment sur la pousse terminale ou sur les rameaux latéraux.

Dès leur prélèvement en forêt ou en pépinière, les boutures placées dans des sacs plastique contenant du coton imprégné d'eau sont transportées rapidement pour la mise en place.

Les boutures ont une longueur de l'ordre de 15 centimètres pour les rejets de souche et de 5 centimètres pour les jeunes plants (les pousses sont alors souvent plus courtes).

Seules les 3 ou 4 feuilles terminales sont conservées. En 1974, les feuilles ont été coupées à moitié, afin de réduire l'encombrement de la bouture. L'apparition de nécroses a fait abandonner cette méthode en 1975. De plus, sur d'autres essences feuillues, Martin et Quillet (1974) ont montré qu'un bon enracinement était souvent lié à une surface foliaire suffisante.

La base humide des boutures est trempée dans un talc contenant des substances de croissance avec ou sans fongicide, sur 1 centimètre environ. Les boutures sont alors immédiatement insérées dans le substrat, à une profondeur de 2 à 4 centimètres. Cette technique de traitement par poudrage a été choisie pour sa facilité de mise en œuvre (Cornu, 1973).

\subsection{Substrat ef disposition des boutures}

Le substrat est constitué par un mélange de tourbe blonde et de gravier de granulométrie 3-8 millimètres (2/1 en volume), contenu dans des bacs plastique perforés à la base. Sauf indication contraire, ce substrat a reçu une fertilisation complète correspondant à la dose optimale pour la culture du chêne et du hêtre sur tourbe (Delran, Garbaye, Le Tacon, 1975).

En 1974, le substrat était en plein. En 1975, chaque bac contenait exactement un jeu de 54 « paper-pots» 7,5 $\times 15$ centimètres contenant le mélange et reposant sur une couche de 5 centimètres du même substrat. Les boutures sont ainsi plus faciles à séparer, à observer, à trier et à transporter en fin d'expérience. Dans tous les cas, un bac contient 54 boutures (écartement moyen 7,5 centimètres).

\subsection{Climat de la serre}

Les bacs sont directement installés sur le sol d'une serre à température automatiquement régulée ( 20 à $25^{\circ} \mathrm{C}$ de jour, 15 à $20^{\circ} \mathrm{C}$ de nuit) et comportant un système de 
refroidissement du type « cooling ». L'humidification des boutures est assurée par une nébulisation intermittante ( 3 minutes $\times 3$ fois/heure en 1974,30 secondes $\times 3$ fois/heure en 1975).

\subsection{Traitements antiparasifaires}

Pour lutter contre le développement de nombreux parasites les boutures furent traitées toutes les semaines:

- oïdium du chêne (alternance soufre micronisé et Karathane),

- pourritures diverses (Captan),

- puceron laineux du hêtre, philaphis fagi (Dimecron 10),

- chenilles (lindane).

\subsection{Dispositifs statistiques}

Chaque essai est disposé selon un plan d'expérience en blocs complets :

- 4 blocs répartis en fonction des différences de climat dans la serre (proximité des parois, force du courant d'air du « cooling »),

- chaque traitement unitaire est constitué par 1 bac de 54 boutures ( 27 de chêne, 27 de hêtre).

La variabilité génétique a été uniformisée entre blocs et traitements par randomisation des nombreux clones (souches ou semis) ; chaque bloc est homogène du point de vue de la date de prélèvement.

L'effet bloc significatif qui apparaît dans certains essais montre l'importance de l'hétérogénéité climatique de la serre ou de la date de prélèvement.

\subsection{Déroulement d'une expérience. Observations et mesures}

Les boutures ont été mises en place entre le 12 mai et la fin juin, suivant l'expérience, l'année et la tardivité du matériel utilisé.

Les mesures sont intervenues après le sevrage (arrêt progressif du mist) entre le 15 août et le 1 er septembre, soit en moyenne après 2 à 3 mois sous nébulisation.

Les boutures devant servir par la suite à d'autres essais concernant leur croissance, il était impossible d'appliquer à la totalité des méthodes d'observation destructives. Les 4 blocs ont donc fait l'objet des complages suivants :

- pourcentage de boutures ayant fait une ou plusieurs pousses depuis leur mise en place (pousses terminales ou latérales),

- pourcentage de boutures présentant une résistance à l'arrachement et que l'on a considérées en première approximation comme enracinées.

Deux à trois semaines plus tard, le bloc no 1 de chaque essai a été arraché dans sa totalité et a fait l'obje! des observations et mesures suivantes :

- pourcentage réel de boutures enracinées,

- nombre de racines par bouture enracinée,

- poids frais de racines par bouture racinée.

Cette méthode destructrice permet d'apprécier l'erreur commise sur l'estimation de l'enracinement par la première méihode (résistance à l'arrachement).

Sur le seul bloc 1 l'estimation est en général supérieure à l'enracinement réel. Celui-ci est donc légèrement surestimé. 
Cette différence est due essentiellement à la présence de gros cals sur certaines boutures non racinées, ce qui crée une résistance à l'arrachement.

Les équations des droites de régression de y (pourcentage réel de boutures racinées, déterminé par arrachage) en fonction de $x$ (pourcentage de boutures résistant à l'arrachement) sont les suivantes (coefficient de corrélation $r=0,86$ pour 20 couples, significatif au seuil de 1 p. 1000 ) :

$$
\begin{aligned}
\text { rejets de souche : } y & =-0,72+0,78 x \\
\text { semis } & y=-5,3+0,88 x
\end{aligned}
$$

\subsection{Interprétation statistique}

La variance des résultats de chaque essai a été analysée selon le modèle des blocs complets, après transformation $y=\operatorname{arc} \sin \sqrt{x}$. Seules ont été interprétées les différences significatives au risque de 5 p. 100.

Sur les figures, un trait horizontal reliant 2 traitements (au-dessus du graphique) indique que les résultats de ces 2 traitements ne diffèrent pas significativement.

\section{Résultats}

\subsection{Observations générales}

\subsubsection{Survie des boutures ef enracinement.}

On obtient une survie de près de 100 p. 100 des boutures dans les meilleurs traitements. Le facteur limitant semble être surtout le stade de développement de la pousse lors du prélèvement (jeune mais suffisamment rigide). Lorsque les boutures meurent, la cause en est toujours une pourriture, qui se produit à la section de la bouture et qui remonte ensuite vers le haut. Les boutures survivantes peuvent faiblement évoluer (cicatrisation de la section), former un gros cal dur au niveau de la section (photo no 1) ou s'enraciner et cicatriser. Les gros cals ont été rarement observées dans

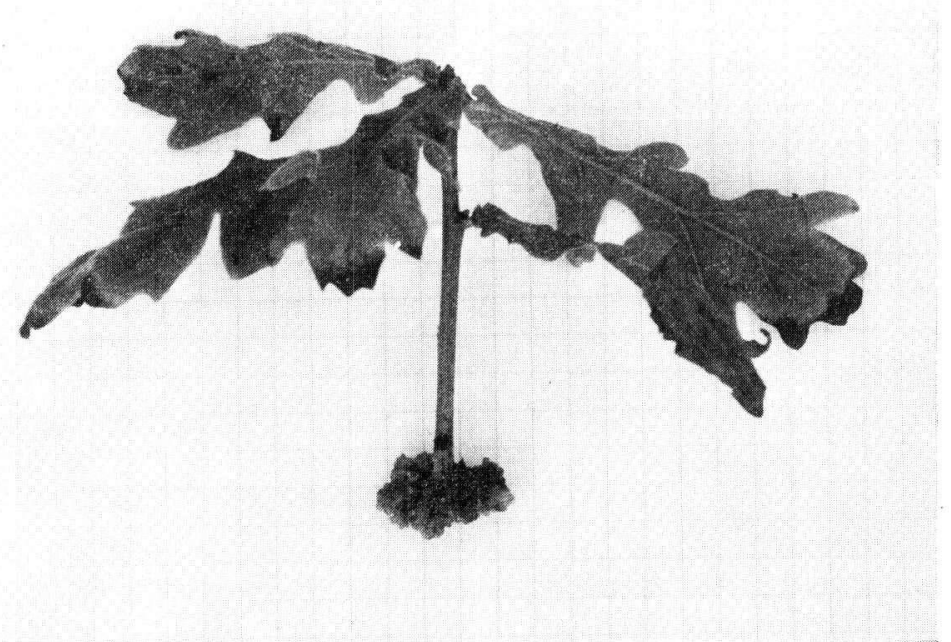

PHOTO No 1. - Bouture de chêne présentant un cal (photo Delran). 
le cas du hêtre (maximum : 10 p. 100 en 1974, dans certains traitements). Pour le chêne, ils étaient abondants en 1974 (jusqu'à 50 p. 100) et rares en 1975, malgré des traitements identiques. Comme la survie des boutures, la formation de gros cals semble être liée au stade physiologique du matériel utilisé (Kleinschmit et al., 1975).

La présence simultanée de racines et d'un gros cal n'a été observée qu'une fois sur plusieurs milliers de boutures.

Dans la suite de l'exposé des résultats, nous ne tiendrons pas compte de la formation de gros cal. Les pourcentages sont trop faibles pour donner des résultats significatifs et nous mettrons l'accent sur la rhizogénèse qui seule a un intérêł pratique.

\subsubsection{Morphologie des racines.}

Les racines apparaissent généralement à l'extrémité de la bouture, ou à l'extrémité inférieure de la partie saine lorsqu'une pourriture s'installe à la base dès le début.

Dans ce dernier cas, la pourriture ne progresse plus dès que la rhizogénèse a eu lieu. Le même phénomène a été observé chez le Douglas par l'un d'entre nous (Cornu, 1973). La rapidité d'enracinement est donc un facteur de succès, puisqu'elle contribue à enrayer la progression de la pourriture.

Ce n'est que lorsque les boutures sont profondément enfoncées dans le substrat (plus de 5 centimètres, pour l'un de nos essais) que des racines peuvent prendre naissance à plusieurs niveaux (photo $n^{\circ} 2$ ), et sur une ou plusieurs génératrices.

Dans le cas général (phoios no 3 et no 4), 1 à 6 racines prennent naissance au niveau de la section de la bouture ( 3 à 4 en moyenne). Très rapidement, elles présentent u ne morphologie très normale pour l'espèce (ramifications, couleur, écorce). En l'absence d'obstacle, la croissance des racines est horizontale (essais de 1974) et la croissance atteint 20 à 30 centimètres en 2 ou 3 mois. En 1975, la présence des « paper-

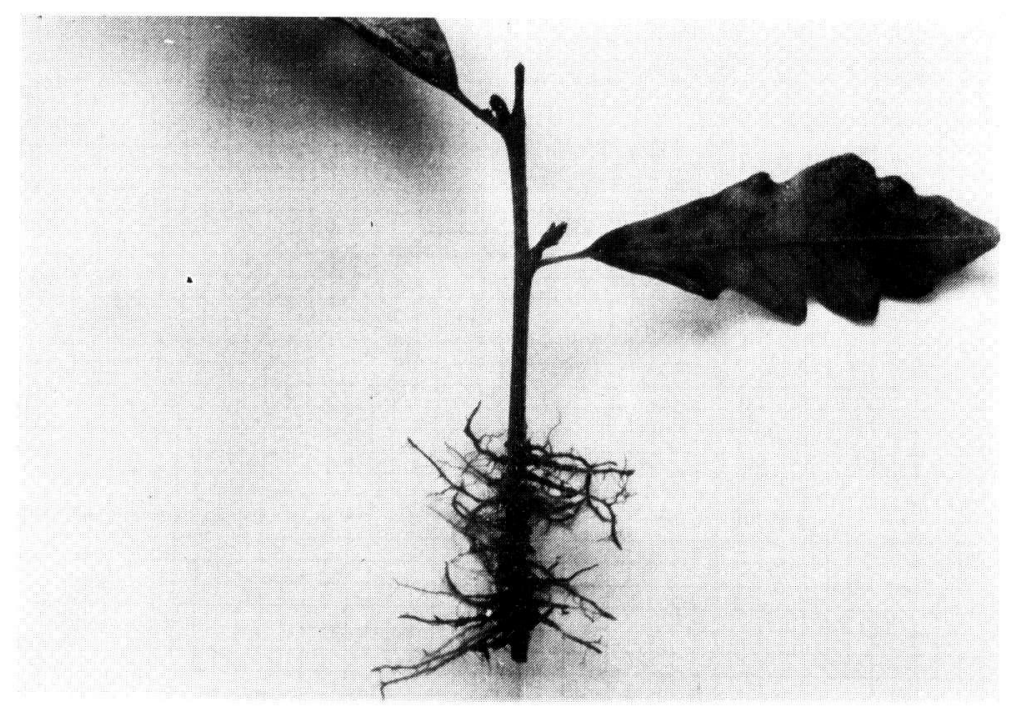

PHOTO No 2. - Bouture de chêne plantée profondément $(5 \mathrm{~cm})$ et présentant des racines à plusieurs niveaux (photo Delran). 
PHOTO No 3. - Bouture de hêtre enracinée après 3 mois de brumisation. Subsirat : tourbe $(2 / 3)$ gravier (1/3). Traitement: talc + 0,5 p. $100 \mathrm{AlB}+15$ p. 100 Captan. Matériel bouturé : pousse de plant de 1 an (photo Delran).
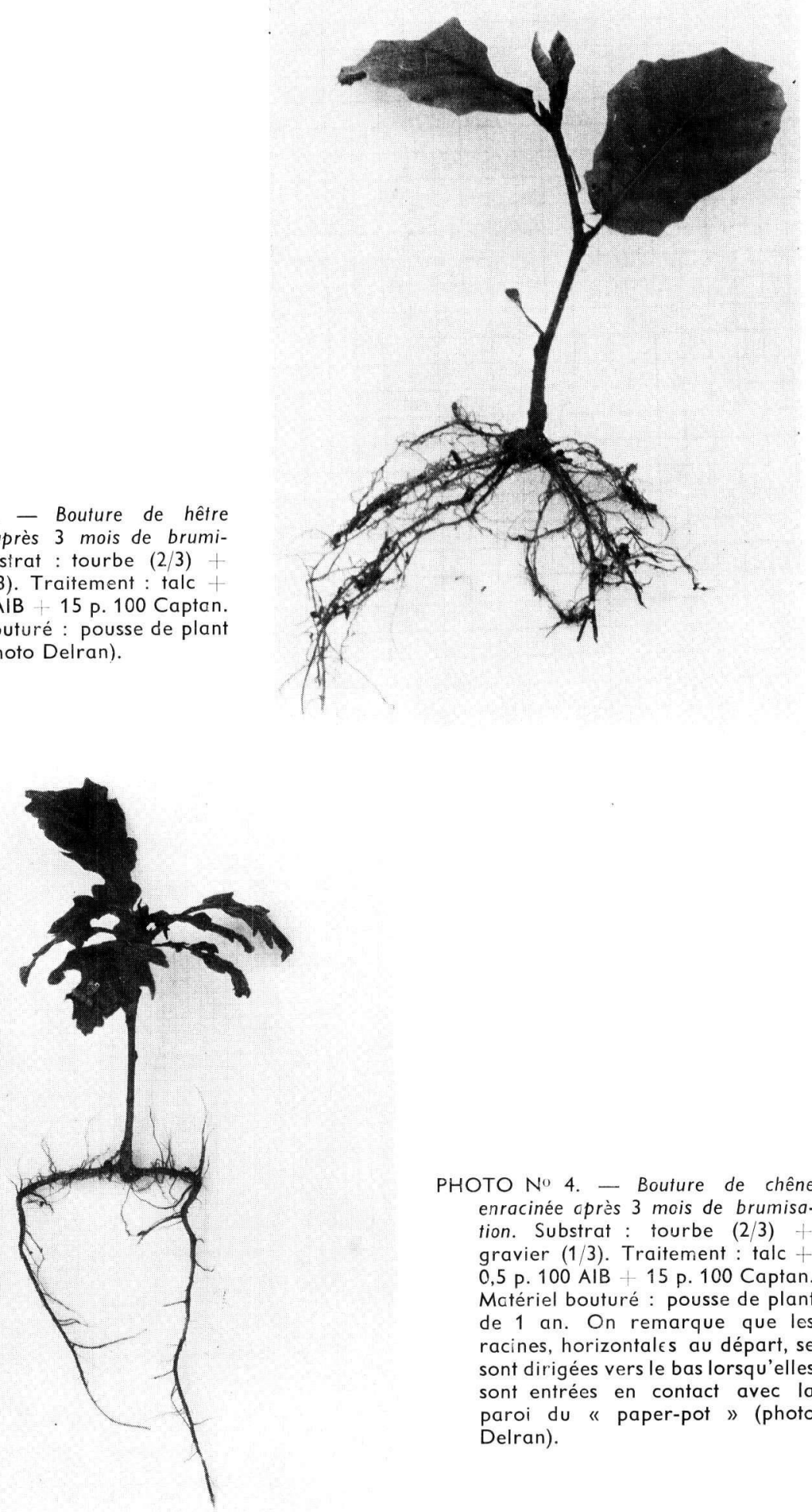

PHOTO No 4. - Bouture de chêne enracinée oprès 3 mois de brumisation. Substrat : tourbe (2/3) gravier (1/3). Traitement: talc + 0,5 p. $100 \mathrm{AlB}+15$ p. 100 Captan. Matériel bouturé : pousse de plant de 1 an. On remarque que les racines, horizontales au départ, se sont dirigées vers le bas lorsqu'elles sont entrées en contact avec la paroi du «paper-pot» (photo Delran). 
pots » entraîne une croissance des racines vers le bas lorsqu'elles rencontrent la paroi de papier (voir photo $\mathrm{n}^{0} 4$ ).

\subsubsection{Développement des bourgeons.}

Le développement des parties aériennes est rarement observée pendant la période de bouturage proprement dite ; elle intervient après le sevrage, surtout dans le cas du chêne. La conception des essais ne permet pas de savoir si le non développement des bourgeons (terminaux ou axillaires) est due à la rhizogénèse ou à la brumisation, mais il y a là un nouvel argument en faveur du sevrage précoce.

\subsection{Boutures de rejets de souche}

3.2.1 Nature du matériel végétal et traitements chimiques.

Cet essai (1975) ne porte que sur le chêne. Afin de permettre un grand nombre de traitements, il a été réalisé dans de petits «paper-pots» $(3 \times 10$ centimètres avec 4 blocs et 15 boutures par traitement unitaire.

Dix traitements chimiques (poudrage) :

$$
\begin{aligned}
& T \text { : talc seul (témoin) Captan : } 25 \text { p. } 100 \\
& \text { AIB : 0,1 p. } 100 \text { (acide Indol Captan : } 25 \text { p. } 100+\text { AIB 0,5 p. } 100 \\
& \text { Butyrique) } \\
& \text { AlB : } 0,5 \text { p. } 100 \\
& \text { Captan : } 25 \text { p. } 100+\text { AIB } 0,1 \text { p. } 100 \\
& \text { AlB : } 1 \text { p. } 100 \\
& \text { Bénomyl : } 15 \text { p. } 100 \\
& \text { AlB : } 2 \text { p. } 100 \\
& \text { Bénomyl : } 15 \text { p. } 100+\text { AlB 0,5 p. } 100
\end{aligned}
$$

Le captan et le bénomyl sont des fongicides destinés à augmenter les chances de survie de la bouture.

Les traitements sans fongicide portent sur deux types de matériel végétal :

- apex de rejets de l'année (non lignifiés),

- apex de rejets de 2 ans.

Les 10 traitements porłent de plus sur des tronçons (2 à 3 feuilles) de rejets de l'année (semi-lignifiés).

La figure 1 montre que, malgré une réussite d'ensemble assez médiocre (maximum d'enracinement : 35 p. 100) due à une surirrigation quelques faits significotifs se dégagent:

- I'AIB à forte concentration a un effet dépressif dans le cas des apex de rejets de 1 an. Au contraire, son effet est très positif pour les rejets de 2 ans et les tronçons semi-lignifiés. L'enracinement du témoin est presque nul. Il y a donc des différences de comportement vis-à-vis des substances de croissance suivant l'état physiologique du matériel, comme cela a déjà été démontré par d'auires auteurs sur des espèces différentes ;

- le bénomyl est le plus efficcce des deux fongicides utilisés. Il a même un effet positif significatif par ropport au témoin, en l'absence d'AIB. En prévenant la pourriture, le bénomyl améliore la survie des boutures et le nombre de boutures qui s'enracinent. 


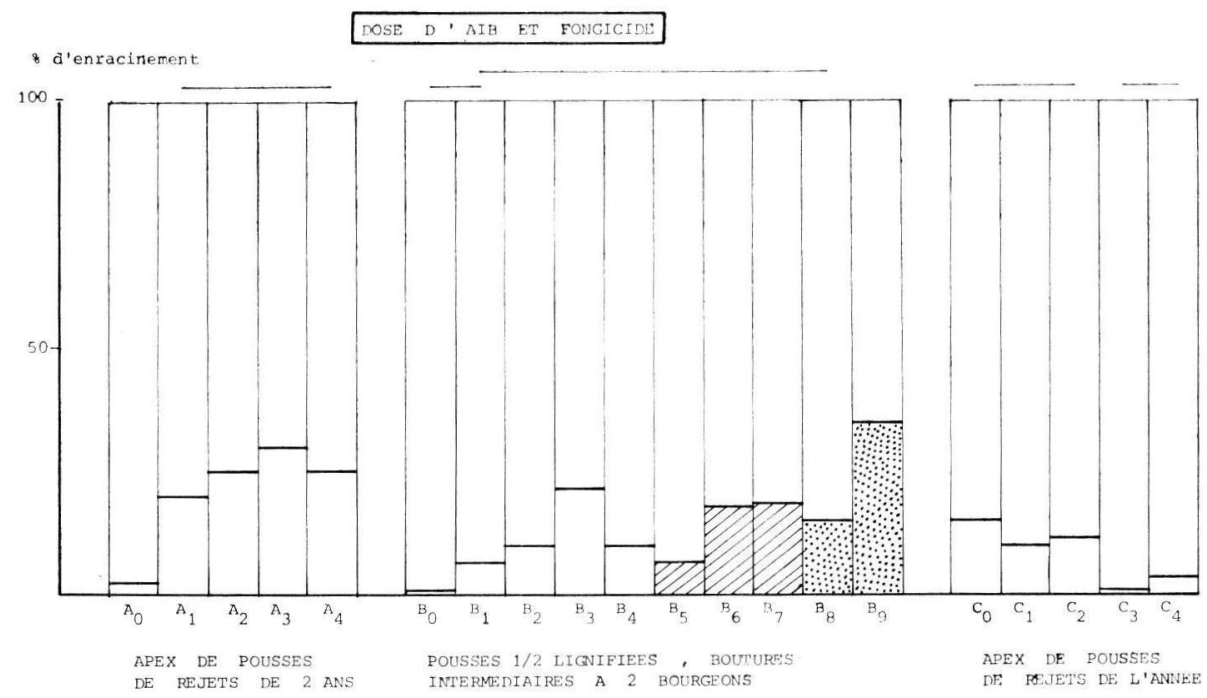

FIG. 1. - Influence du traitement chimique sur le taux d'enracinement. Boutures de rejets de souche de chêne. Détail des traitements : 0 : talc pur. 1 : talc $+0,1$ p. 100 AlB. 2 : talc + 0,5 p. 100 AIB. 3 : talc +1 p. 100 AlB. 4 : talc +2 p. 100 AlB. 5 : talc +25 p. 100 Captan. 6 : talc +25 p. 100 Captan + 0,5 p. 100 AlB. 7 : talc + 25 p. 100 Captan + 0,1 p. 100 AlB : 8 talc + 15 p. 100 Benomyl. 9 : talc +15 p. 100 Benomyl $+0,5$ p. 100 AlB. Hachures : Captan. Pointillés : Benomyl.

Un essai réalisé en 1974 montrait qu'à la dose de 0,5 p. 100 et sans fongicide, I'AIA (acide indolylacétique) et I'AIB avaient la même efficacité pour le chêne (jeunes plants ou rejets de souche), mais que I'AIB était nettement préférable pour le hêtre et permettait d'atteindre 90 p. 100 de réussite avec des rejets de souche. L'AlB, de conservation plus facile, a été retenu dans tous les cas pour nos expériences futures.

3.2.2 Propriétés physiques du substrat (essai réalisé en 75).

Quatre substrats différents ont été expérimentés :

- tourbe blonde pure (T),

- 2/3 (en volume) de tourbe $+1 / 3$ de gravier 3-8 millimètres (TG),

- 2/3 de gravier $+1 / 3$ de tourbe (GT),

- gravier pur (G).

Aucune fertilisation n'a été appliquée. Traitement chimique : 0,5 p. 100 AIB 15 p. 100 coptan.

La figure 2 montre que les pourcentages d'enracinement sont plus faibles avec les traitements $G$ et GT. Ceite différence n'est significative que dans le cas du hêtre.

D'autre part, la composition du substrat n'a pas d'effet sur le poids moyen de racines par bouture, bien que le nombre de racines par bouture soit significativement supérieur sur le gravier pur.

Nous refiendrons donc la tourbe pure comme étant le meilleur substrat et le plus facile à mettre en œuvre (aucun mélange n'est nécessaire, la manutention est facilitée par le faible poids des bacs, ef la formation d'une motie évite de léser les racines au repiquage). Ceci est contraire à ce que trouvent d'autres auteurs (notamment Kleinschmit et al., 1975) qui expliquent la supériorité du gravier ou du sable grossier par 

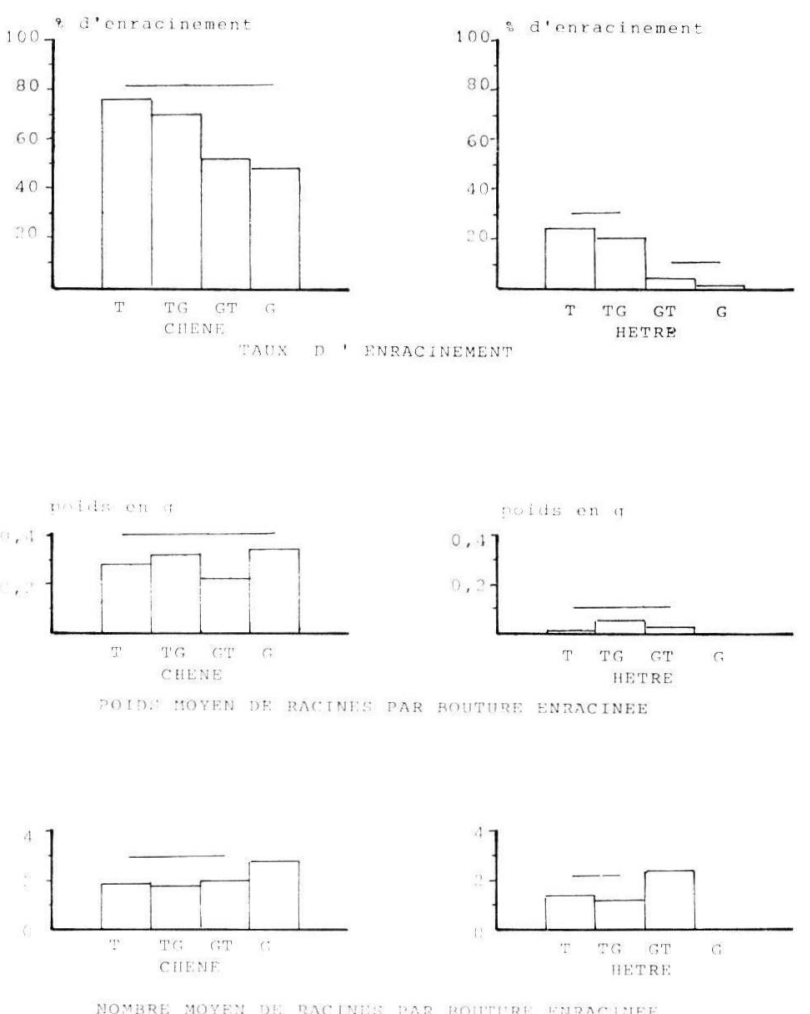

FIG. 2. - Influence des propriétés physiques du substrat sur le taux d'enracinement, le poids ef le nombre moyen de racines par bouture enracinée. Boutures de rejets de souche. Traitement chimique : talc $+0,5$ p. 100 AIB +15 p. 100 Captan. Pas de fertilisation du substrat.

une meilleure aération de la base des boutures. II est donc possible que les résultats dépendent de l'intensité et du rythme de la nébulisation. Nous prévoyons un essai dans ce sens en 1976. Néanmoins, à valeur égale du point de vue de la rhizogénèse, la tourbe à l'avantage d'avoir toutes les caractéristiques d'un bon substrat pour la croissance ultérieure des plants, sans repiquage (forte capacité en eau utile, forte capacité d'échange cationique permettant l'apport d'engrais). Le système tourbe + « paperpot» devrait donc simplifier au maximum l'obtention de plants à partir de boutures.

\subsubsection{Propriétés chimiques du substrat (essais réalisés en 1974 et 1975).}

Les essais combinent $3 \mathrm{pH}(\mathrm{pH} 4, \mathrm{pH} 6$ et $\mathrm{pH} 8$, obtenus par apport de carbonate de calcium) et 3 doses de fertilisation (0,2 et 8). La dose de base (dose 1) correspond à 150 grammes de $\mathrm{N} /$ mètre cube, 150 grammes $\mathrm{P}_{2} \mathrm{O}_{5} /$ mètre cube et 150 grammes $\mathrm{K}_{2} \mathrm{O}$ /mètre cube, plus les éléments suivants : $\mathrm{S}, \mathrm{Ca}, \mathrm{Mg}, \mathrm{Fe}, \mathrm{Mn}, \mathrm{Cu}, \mathrm{Zn}, \mathrm{B}, \mathrm{Mo}$.

Nous avons par ailleurs montré que la dose 2 était suffisante pour assurer une croissance excellente des semis de chêne et de hêtre (Delran, Garbaye, Le Tacon, 1975). 
En 1975, l'essai comportait un traitement supplémentaire : tourbe pure (dose 0. $\mathrm{pH}$ 4), avec deux pulvérisctions foliaires de solution nutritive du commerce (Quinoligo) par semaine.

Pour les deux années d'expérimentation, il n'est apparu aucun effet significatif des différents traitements. Provisoirement, nous retiendrons donc la seule conclusion possible : la fertilisation du substrat, aux doses susceptibles d'assurer le développement ultérieur des boutures racinées, n'est pas un obstacle à la rhizogénèse.

\subsection{Boutures de jeunes plants}

\subsubsection{Influence du recépage (1975).}

Des plants de 1 an de 40 centimètres de hauteur élevés sur tourbe fertilisée, ont fait l'objet de deux traitements :

- pas de recépage,

- recépage à 10 centimètres du sol, à la fin de l'hiver. Pour les deux espèces, les pousses obtenues après recépage provenaient de bourgeons dormants sur le bas de la tige ou des bourgeons de petits rameaux préexistants.
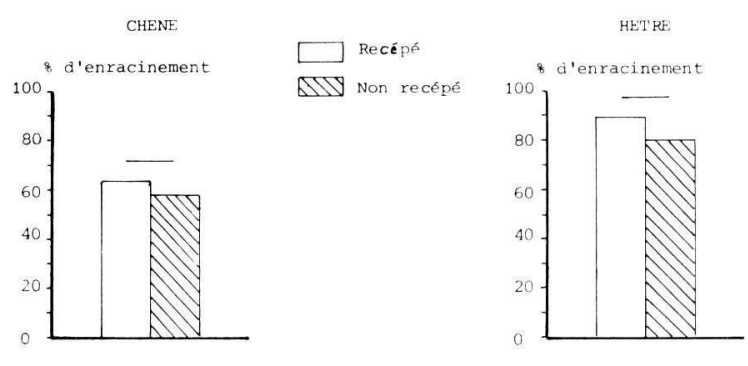

TAUX D 'ENRA' INEMENT
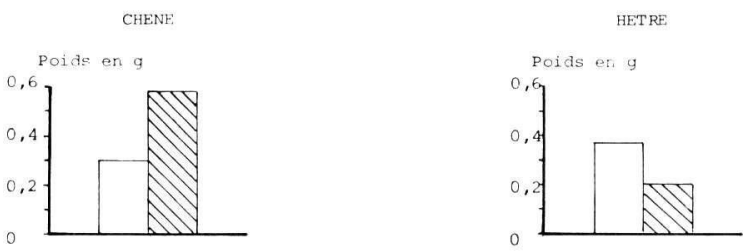

POIDS MOYEN DES PACINES PAR BOITURE ENRACINEE

CHENE
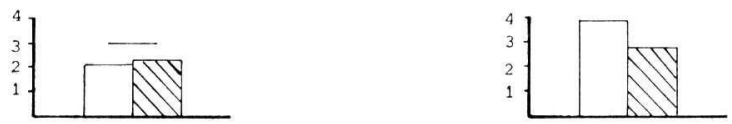

NOMBRE MOYEN Dï RACINES PAR BOUTURE ENRACINEE

FIG. 3. - Influence du recépage du pied-mère - plant de 1 ans - sur le taux d'enracinement, le poids ef le nombre moyen de racines par bouture enracinée. Traitement chimique : 0,5 p. 100 AlB. 
Traitement chimique : 0,5 p. 100 AlB.

La figure 3 montre que, pour les deux espèces, le traitement ne compromet pas la réussite du bouturage : il n'y a aucun effet significatif.

Dans le cas du chêne, les boutures provenant des plants recépés présentent un poids moyen de racine par bouture enracinée inférieur de moitié à celui des boutures provenant des plants non recépés, sans qu'il y ait d'effet sur le nombre de racines par bouture enracinée.

Chez le hêtre, par contre, le recépage augmente le poids moyen de racines par bouture enracinée et le nombre de racines par bouture enracinée.

Le recépage des plants, sans jouer sur le taux de réussite de bouturage, améliore donc la qualité de l'enracinement du hêtre et diminue celle du chêne.

3.3.2 Influence de la nutrition des pieds-mères (1975).

La fertilisation ef la nutrition des pieds-mères modifient-elles l'aptitude des boutures à l'enracinement?

Nous avons prélevé des boutures sur des plants de 1 an élevés sur tourbe à différents $\mathrm{pH}$ et à différents niveaux de fertilisation :

$$
\left.\left.\begin{array}{l}
-\mathrm{pH} \mathrm{3,6} \\
-\mathrm{pH} \mathrm{5,5} \\
-\mathrm{pH} 7
\end{array}\right\} \text { dose } 4 \quad \begin{array}{l}
- \text { dose } 6 \\
\\
- \text { dose } 1 \\
- \text { dose } 4 \\
- \text { dose } 8
\end{array}\right\} \mathrm{pH} \mathrm{3,6}
$$

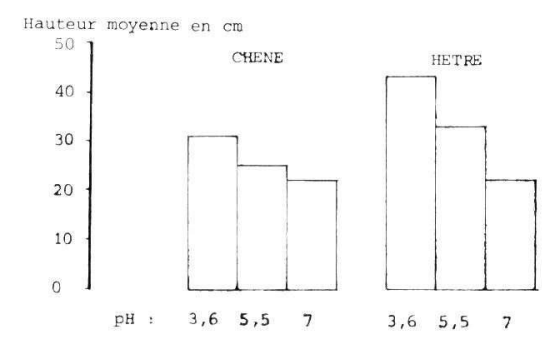

ase constante (dose 4)

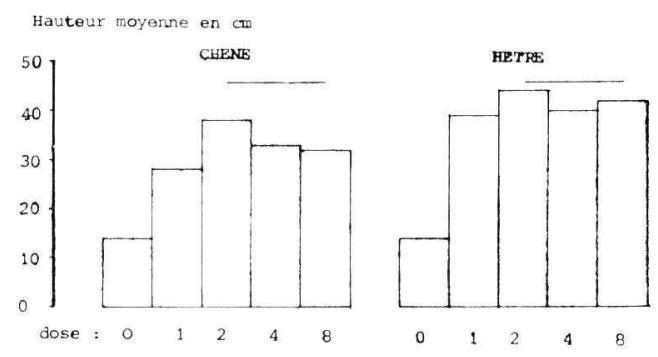

a $\mathrm{pH}$ constant $(\mathrm{pH} 3,6)$

FIG. 4. - Hauteur moyenne des pieds-mères en fonction $\mathrm{du} \mathrm{pH}$ de la tourbe ef de la dose de fertilisation (semis de 1 an). 
Les hauteurs moyennes des plants en fin de 1 re année sont données par la figure 4, qui montre que les meilleures croissances sont obtenues pour les 2 espèces avec les $\mathrm{pH}$ les plus bas et à partir de la dose 2 .

Les boutures traitées par l'AIB 0,5 p. 100 ont donné les résultats présentés dans la figure 5 .
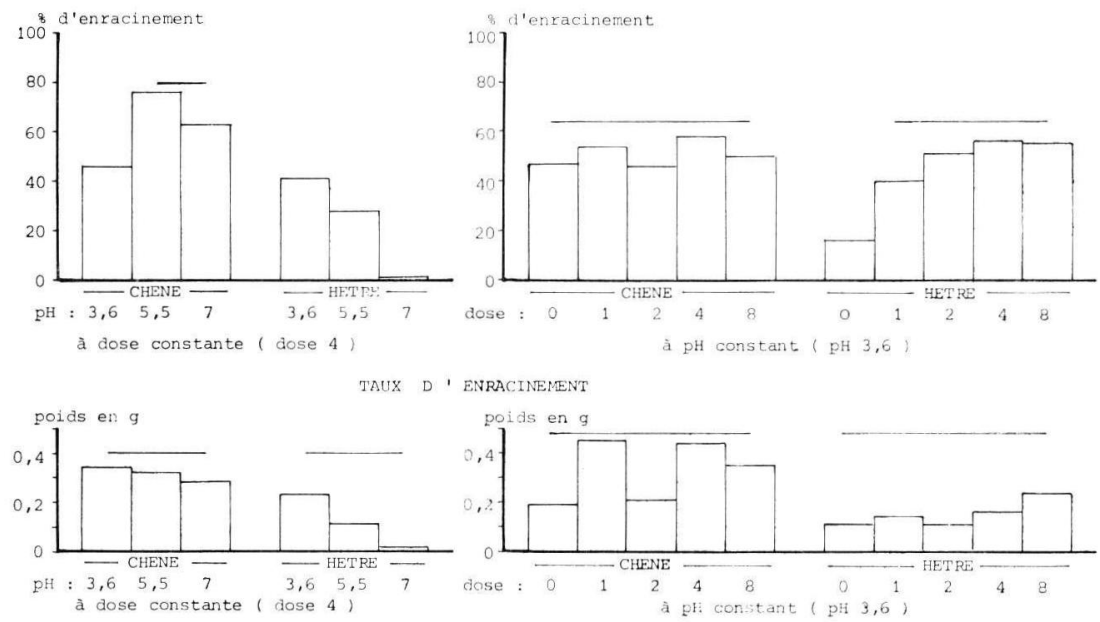

POIDS MOYEN DE RACINES PAR BOUTUPE ENRACINEE

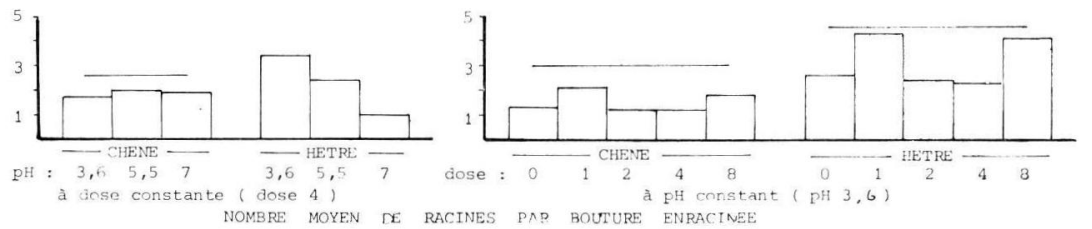

FIG. 5. - Influence de la fertilisation du pied-mère sur le taux d'enracinement, le poids moyen et le nombre moyen de racines par bouture enracinée. Boutures de plants de 1 an. Traitement chimique : talc $+0,5$ p. 100 AlB.

Dans le cas du hêtre, on note une très bonne concordance entre la vigueur des plants exprimée par leur hauteur moyenne et l'aptitude des boutures à l'enracinement ; l'influence du pH est particulièrement nette : 40 p. 100 d'enracinement pour les plants élevés à $\mathrm{pH}$ 3,6 et 2 p. 100 pour les plants élevés à $\mathrm{pH} 7$.

Pour le chêne, les résultats sont moins nets et différents : pas d'effet significatif de la dose de fertilisation, et action du $\mathrm{pH}$ inverse de ce que l'on observe pour le hêtre les meilleurs résultats étant obtenus pour les $\mathrm{pH}$ élevés (alors que la hauteur des semis est plus grande pour les bas $\mathrm{pH}$, comme dans le cas du hêtre).

Le poids moyen de racines par bouture enracinée ne présente de différences significatives que dans le cas des variations de $\mathrm{pH}$ pour le hêtre, dans le même sens que le pourcentage de boutures racinées. Le nombre moyen de racines par bouture enracinée ne présente pas de variations significatives d'un traitement à l'autre. 


\subsubsection{Influence du traitement hormonal (1974).}

La comparaison des traitements AIB 0,5 p. 100 et AIA 0,5 p. 100 ne révèle aucune différence d'efficacité des deux substances de croissance (pousses prélevées sur des semis naturels de 5 ans, en forêt).

\subsubsection{Influence des propriétés chimiques du substrat (1974).}

Les conclusions sont les mêmes que dans le cas des boutures de rejets de souche ; nous n'avons pas obtenu d'effet significatif $\mathrm{du} \mathrm{pH}$ et de la fertilisation du substrat sur l'enracinement des boutures.

\section{Discussion et conclusion}

Si nous comparons nos résultats à ceux de Kleinschmit et al. (1975), qui ont réalisé l'étude la plus complète et la plus récente dans le domaine du bouturage du chêne, un certain nombre de différences apparaissent : pour nous, la tourbe pure est le meilleur substrat, alors que le gravier pur est de loin supérieur pour Kleinschmit et al.

Nous avons d'autre part obtenu d'excellents taux d'enracinement (80 à 90 p. 100) pour des boutures prélevées en 1974 sur des semis de chêne de plus de 5 ans, alors que pour les auteurs allemands le taux d'enracinement s'effondre après 2 à 3 ans.

On observe d'ailleurs très fréquemment des divergences suivant les auteurs (voir revue bibliographique de Kleinschmit et al. au début de leur article); elles sont dues à la multiplicité des facteurs en cause, dont certains sont ignorés et les autres imparfaitement et empiriquement contrôlés (température, humidité, nature génétique du matériel, stade physiologique, etc...). Les interactions possibles entre ces facteurs contribuent à rendre les interprétations complexes. Le type de recherches systématiques combinant un grand nombre de facteurs choisi par Kleinschmił et al. est le plus susceptible de faire progresser l'aspect fondamental des connaissances, justement parce qu'il met en évidence les interactions. En particulier, il ressort de leurs travaux qu'on peut réduire l'influence d'un facteur (date de prélèvement ou âge du pied-mère, par exemple) en utilisant un mélange de substances de croissances. Il est à noter que la concentration totale optimale du talc en substances de croissance reste de l'ordre de 0,5 p. 100 , ce que nous avons retenu pour l'AIB dans nos propres expériences.

Notre démarche a ceci de différent, que dans un premier temps, nous avons mis l'accent sur les problèmes de nutrition minérale (des pieds mères et des boutures) et de réalisation pratique d'un programme de multiplication (bouturage d'arbres âgés, traitement des pieds-mères).

En ce qui concerne les 2 espèces que nous avons étudiées, nos résultats les plus significatifs sur le plan pratique sont les suivants :

- Il est possible d'obtenir 80 à 90 p. 100 de boutures racinées de chêne ou de hêtre, soit à partir de jeunes plants, soit à partir d'arbres adultes recépés.

- Le fait de pouvoir bouturer des rejets de souche d'arbres âgés ( 90 ans) est un acquis primordial pour la constitution d'une tête de clone : à cet âge, on peut déjà juger valablement un phénotype de chêne ou de hêtre (forme, qualité du bois). La voie suivie par Kleinschmit ef al. (gourmands au lieu de rejets de souche) n'a pas conduit à des résultats intéressants. Les gourmands de la base du tronc (donc proche du collet) donnant néanmoins les taux d'enracinement les plus élevés. 
- Dans le cas du hêtre, la fertilisation et la vigueur des pieds-mères en pépinière a un effet primordial. Les bonnes conditions de croissance (bas $\mathrm{pH}$ de la tourbe utilisée comme substrat, forte fertilisation) favorisent l'enracinement des boutures. Ceci est d'autant plus intéressant qu'un plant vigoureux produit plus de boutures.

- Nous disposons déjà de certains éléments concernant le traitement et la gestion d'une pépinière de pieds-mères : l'aptitude au bouturage est encore bonne jusqu'à plus de 5 ans, ef le recépage ne présente aucun inconvénient, pour le chêne comme pour le hêtre.

- Enfin, la tourbe pure, dans nos propres conditions de travail, est un bon substrat : l'enracinement des boutures est au moins aussi bon que dans le gravier, et les manipulations ultérieures sont facilitées (repiquage sans léser les racines grâce à la motte, ou fertilisation avec repiquage différé).

De nombreux points sont encore à étudier :

- croissance ultérieure des plants issus de bouture ;

- recherche du stade physiologique assurant le meilleur enracinement (critères morphologiques et anatomiques);

- stimulation du rejet de souches;

- recherches des causes du comportement différent du chêne et du hêtre.

Tous ces points sont en cours d'étude et certains feront l'objet d'une prochaine publication.

Reçu pour publication en décembre 1976.

\section{Summary}

Looking after the best rooting conditions of herbaceous cuttings of oak (Quercus petraea (M.) Liebl.) and beech (Fagus silvatica L.)

Vegetative propagation of oak and beech could improve the technics of artificial regeneration and the quality of the stands of these fwo species in France.

After two years of investigations on mist propagation of oak and beech, we have obtained the following results (cuttings sampled in may and june, rooting period of about 3 months, in green-house) :

- it is possible to obtain 80 to 90 p. 100 of rooted cuttings of both species, from young mother stocks in the nursery or from stumpshoots of mature trees after felling ;

- the best results are obtained by powdering the lower end of the cutting with talc $+0,5$ p. 100 IBA +15 p. 100 Benomyl ;

- the mineral nutrition and growth rate of the beech mother stocks in the nursery bave a great importance ;

- the rooting ability is still good with 5 years old mother stocks, and the cutting down of the stocks does not reduce the rooting ability of the shoots ;

- pure sphagnum peat is a good medium for the rooting of the cuttings

\section{References bibliographiques}

CORNU D., 1973. Essais préliminaires sur la sélection de clones bouturables de Douglas (Pseudotsuga menziezii Franco) Ann. Sci. forest., 30 (2).

CORNU D., GARBAYE J., LE TACON F., 1975. Résultats d'un essai préliminaire sur le bouturage du chêne et du hêtre R.F.F., XXVIII, 2.

DELRAN S., GARBAYE J., LE TACON F., 1976. Production rapide de plants feuillus sur tourbe fertilisée. R.F.F., XXVII.

FLEMMER W., 1962. The vegetative propagation of oaks. Proc. Internat. PI. Propag. Soc., 12. 
GARBAYE J., LE TACON F., MORMICHE A., 1976. Les techniques de production de plants de chêne et de hêtre en France : situation actuelle, orientation des recherches en cours et application. Bulletin Technique de l'Office National des Forêts, no 9.

KLEINSCHMIT J., WITTE R., SAUER A., 1975. Möglichkeîen der züchterischen Verbesserung von Stiel- und Traubeneichen (Quercus robur und Quercus petraea). II. Versuche zur Stecklingsvermehrung von Eiche. Allg. Forst u. J. Zig., 146, Jg, 10.

LE TACON F., MORMICHE A., 1974. A propos de l'écologie du chêne de tranchage en Lorraine. Bulletin technique de l'Office National des Forêț, $n^{\circ} 6$.

MARTIN B., 1974. Bouturage des arbres forestiers au Congo. Bois et forêts des Tropiques, nos 154 , 155,156 et 157.

MUHLE-LARSEN C., 1946. Experiments with Softwood cuttings of forest trees. Det Forstlige Forsögsvaesen i Danmark (17).

POLGE H., 1973. Etat actuel des recherches sur la qualité du bois de hêtre. Bullatin technique de l'Office National des Forêts, $n^{0} 4$.

POLGE H., KELLER R., 1973. Qualité du bois et largeur d'accroissements en forêt de Tronçais. Ann. sci. forest., 30 (2).

THIMANN K. V., BEHNKE-ROGERS J., 1950. The use of auxins in the rooting of woody cuttings. Maria Moors Cabot. Foundation, pub. no 1.

VIEITEZ E., 1974. Vegetative propagation of Chestnut. N.Z.J. For. Sci., 4 (2). 